\title{
Analysis of the stability of InGaN/GaN multiquantum wells against ion beam intermixing
}

\author{
A. Redondo-Cubero ${ }^{1,2^{*}}$, K. Lorenz ${ }^{1}$, E. Wendler ${ }^{3}$, S. Magalhães ${ }^{1}$, E. Alves ${ }^{1}$, D. \\ Carvalho $^{4,5}$, T. Ben ${ }^{4,5}$, F.M. Morales ${ }^{4,5}$, R. García ${ }^{4,5}$, K.P. O’Donnell ${ }^{6}$, C. Wetzel ${ }^{7}$ \\ 1 Instituto Superior Técnico, Universidade de Lisboa, Estrada Nacional 10, 2695-066 Bobadela LRS, \\ Portugal \\ 2 Departamento de Física Aplicada y Centro de Microanálisis of Materiales, Universidad Autónoma de \\ Madrid, E-28049 Madrid, Spain \\ 3 Friedrich-Schiller-Universität Jena, Institut für Festkörperphysik, Max-Wien-Platz 1, 07743 Jena, \\ Germany \\ 4 Department of Materials Science and Metallurgic Engineering, and Inorganic Chemistry, Faculty of \\ Sciences, University of Cadiz, Spain \\ 5 IMEYMAT, Institute of Research on Electron Microscopy and Materials, University of Cádiz, Spain \\ 6 SUPA Department of Physics, University of Strathclyde, Glasgow, G4 0NG, Scotland,UK \\ 7 Department of Physics, Applied Physics, and Astronomy, Rensselaer Polytechnic Institute, 110 Eighth \\ Street, Troy, New York 12180-3590 USA
}

Ion-induced damage and intermixing was evaluated in InGaN/GaN multi-quantum wells (MQWs) using $35 \mathrm{keV} \mathrm{N} \mathrm{N}^{+}$implantation at room temperature. In-situ ion channeling measurements show that damage builds up with a similar trend for In and Ga atoms, with a high threshold for amorphization. The extended defects induced during the implantation, basal and prismatic stacking faults, are uniformly distributed across the quantum well structure. Despite the extremely high fluences used (up to $4 \times 10^{16} \mathrm{~cm}^{-2}$ ), the InGaN MQWs exhibit a high stability against ion beam mixing.

\footnotetext{
* Corresponding author. E-mail: andres.redondo@uam.es
} 


\section{Introduction}

InGaN/GaN multiple quantum wells (MQWs) are the basis of many modern optoelectronic devices, including bright light emitting diodes, laser diodes, light displays, solar cells, etc. However, the strain induced by the lattice mismatch, in addition to the spontaneous polarization in III-N heterostructures, affects the internal quantum efficiency through the so-called quantum-confined Stark effect (QCSE), which induces a spatial separation of electron and hole wavefunctions ${ }^{1}$, in particular for high InN-content devices, with wide MQW emitting in the green spectral region. One of the strategies suggested to mitigate QCSE in GaN-based materials is the fabrication of intermixed or graded quantum structures by ion beam mixing ${ }^{2,3}$. This approach has been successfully applied in $\mathrm{AlGaAs} / \mathrm{GaAs}{ }^{4,5}, \mathrm{AlGaInP} / \mathrm{GaInP}^{6}$ and $\mathrm{MgZnO} / \mathrm{ZnO}{ }^{7}$ multiquantum wells (MQWs), but it remains unexplored in InGaN/GaN systems.

Despite the outstanding radiation resistance of GaN (with amorphization thresholds above 10 displacements per atom), recent experimental results in GaN/AIN MQWs ${ }^{8}$ have proved that partial intermixing can be induced at low temperatures $(15 \mathrm{~K})$ for sufficiently high Ar fluences. This intermixing induced by collision cascades was shown to be more efficient than thermal interdiffusion ${ }^{8}$, and motivates the present studies. We consider here conventional $\mathrm{InGaN} / \mathrm{GaN}$ nanostructures under more adaptable conditions for industrial scalability, i.e., room temperature implantation with native ions. Thus, in this work we investigate the damage build-up during ion mixing processes in $\mathrm{InGaN} / \mathrm{GaN}$ MQWs with $35 \mathrm{keV} \mathrm{N} \mathrm{N}^{+}$ions, comparing the behavior with thick InGaN layers.

\section{Experimental}


The $\operatorname{In}_{\mathrm{x}} \mathrm{Ga}_{1-\mathrm{x}} \mathrm{N} / \mathrm{GaN}$ MQW sample was grown by metal-organic chemical vapor deposition on a (0001) GaN/sapphire template ${ }^{9}$. The active layers consist of $5 \operatorname{In}_{\mathrm{x}} \mathrm{Ga}_{1}$ ${ }_{\mathrm{x}} \mathrm{N} / \mathrm{GaN}$ periods with thicknesses of $3 \mathrm{~nm} / 20 \mathrm{~nm}$ (measured by transmission electron microscopy) and an average $\mathrm{InN}$ molar fraction in the QWs of $x=10 \%$ (determined by Rutherford backscattering spectrometry, RBS). A $\sim 20 \mathrm{~nm}$ GaN capping layer was deposited to protect the active layers. Photoluminescence and ionoluminesce measurements reveal a green QW emission centered at $\sim 530 \mathrm{~nm}(2.34 \mathrm{eV})$.

Ion implantation and in-situ RBS in channeling mode (RBS/C) studies were carried out in the double-beam chamber at the Institut für Festkörperphysik (Jena) ${ }^{10}$. The implantations were performed at room temperature with $35 \mathrm{keV} \mathrm{N}^{+}$ions, being the energy selected to place the maximum of the damage profile in the central region of the MQWs. According to Monte Carlo simulations with the TRIM code the mean $\mathrm{N}$ range is $55 \mathrm{~nm}$, with a longitudinal straggling of $25 \mathrm{~nm}^{11}$. The ion fluence was increased sequentially from $5 \cdot 10^{12}$ up to $4 \cdot 10^{16} \mathrm{~cm}^{-2}$ and the sample was tilted by $7^{\circ}$ off-axis during the implantation to avoid channeling effects. After each implantation step, the damage accumulation was evaluated in-situ by RBS/C spectra of the sample using a 2.2 $\mathrm{MeV} \mathrm{He}$ collimated beam $\left(\sim 1 \mathrm{~mm}^{2}\right)$. RBS/C spectra were taken in the $<0001>$ aligned direction, using a 3-axis goniometer for sample orientation.

The final as-implanted sample, corresponding to a total fluence of $4 \cdot 10^{16} \mathrm{~cm}^{-2}$, was further studied ex-situ with X-ray diffraction (XRD), transmission electron microscopy (TEM), and grazing incidence RBS. XRD data were acquired in a D8Discover high resolution diffractometer (Bruker-AXS) using $\mathrm{Cu}\left(\mathrm{K}_{\alpha 1}\right)$ radiation, an asymmetric twobounce $\mathrm{Ge}(220)$ monochromator, and a $\mathrm{NaI}$ scintillation detector. To reduce the 
divergence, the incoming X-ray beam is collimated with a $0.2 \mathrm{~mm}$ slit while the diffracted beam is collimated with a $0.5 \mathrm{~mm}$ motorized slit and a $0.1 \mathrm{~mm}$ fixed slit, being the final $2 \theta / \omega$ reproducibility $\pm 0.0001^{\circ}$. XRD $2 \theta / \omega$ curves were simulated using the dynamical theory of X-ray diffraction ${ }^{12}$. The influence of the implantation is taken into account by including the Debye-Waller parameter on the structure factor of individual layers, thus attenuating the derived intensity and considering a $c$-lattice expansion following the work of Boulle and Debelle ${ }^{13}$.

TEM experiments were carried out using a JEOL 2010F microscope operated at 200 $\mathrm{kV}$. Structural and compositional features were studied from micrographs collected by HR-TEM and high angle annular dark field (HAADF) imaging in scanning-TEM (STEM) mode, while nanoprobe analyses of local proportions of atomic elements were based upon energy dispersive X-ray spectroscopy done in the same mode (EDXSTEM). Grazing incidence RBS experiments were carried out with a $2 \mathrm{MeV} \mathrm{He}^{+}$beam using the Van de Graaff accelerator at CTN/IST (Portugal).

\section{Results and discussion}

Fig. 1a shows RBS/C spectra of the sample after the implantation to different total $\mathrm{N}$ fluences. Remarkably, the In signal does not show any shift to higher energies even for the highest fluence, what confirms that there is neither appreciable diffusion of In towards the surface, nor significant sputtering of the GaN capping layer. Two energy windows were defined for $\operatorname{In}\left(\mathrm{w}_{1}\right)$ and $\mathrm{Ga}\left(\mathrm{w}_{2}\right)$ at equivalent depths corresponding to the central region of the damage profile in the MQWs and avoiding the surface peak zone. The minimum yield $\left(\chi_{\mathrm{min}}\right)$, determined as the ratio of the aligned and the random backscattering yield, was obtained for both windows. The increase of $\chi_{\text {min }}$ with fluence 
determines the damage accumulation. The damage level is then described as the difference in the minimum yield of the implanted and virgin sample, $\Delta \chi_{\min }=$ $\chi_{\min }\left(\right.$ implanted) $-\chi_{\min }\left(\right.$ virgin). As a reference, the initial $\chi_{\min }$ values for the as-grown sample were $4.4(1) \%$ and $2.2(1) \%$ for In and $\mathrm{Ga}$, respectively. For the maximum fluence of $4 \cdot 10^{16} \mathrm{~cm}^{-2}$ the aligned level almost overlaps with the random level $\left(\chi_{\min }=100 \%\right)$, indicating a level of damage that is maximal for measurement by RBS/C.

Fig. 1b shows the damage level obtained for $\operatorname{In}\left(\mathrm{w}_{1}\right)$ and $\mathrm{Ga}\left(\mathrm{w}_{2}\right)$ for the entire range of fluences. Both curves have a similar behavior in two main regions of interest. For low fluences $\left(<10^{15} \mathrm{~cm}^{-2}\right)$, damage builds up continuously but the damaged fraction remains below 4\%. The slowing down of damage accumulation, here occurring for fluences between $10^{14} \mathrm{~cm}^{-2}$ and $10^{15} \mathrm{~cm}^{-2}$, is typical of materials with strong dynamic annealing $^{14}$. In GaN films implantation damage in this low fluence regime is characterized by the formation of small point defect clusters and low concentrations of stacking faults, while for higher fluences larger defect clusters form, including extended defects such as stacking faults ${ }^{15}$. Fig. $1 \mathrm{~b}$ shows a strong increase of damage level for fluences above $10^{15} \mathrm{~cm}^{-2}$ which we attribute to the formation of stable defect clusters and extended defects.

In general, the damage build-up curve for the InGaN MQW is qualitatively similar to those reported by Kucheyev et al. and Wendler et al. for gold implantation at $\operatorname{low}^{16}$ and room $^{14}$ temperature, included in Fig. 1 as references. Both In and Ga show the same trend, but the damage accumulation for Ga appears to be systematically lower. This agrees with previous experiments in thin $\mathrm{InGaN}$ films, which have also shown similar levels of damage build-up for the In- and Ga-sublattice, but where a significant higher resistance was found for $\mathrm{GaN}$ than for $\mathrm{InGaN}$, even for very small amounts of $\mathrm{InN}^{14,16}$. 
Solid lines in Fig. 1b represent the fits from Hecking's model, which takes into account the production $\left(P_{p d}\right)$, recombination $\left(R_{p d}\right)$, and clustering $\left(C_{p d}\right)$ of point defects in semiconductors, as well as the production of amorphous regions $\left(P_{a}\right)$ and their growth $\left(G_{a}\right)^{17,18}$. The results obtained from these fits are summarized in Table 1. The variations observed with the values obtained from Wendler et al. ${ }^{16}$, in particular the low values of $P_{a}$ obtained here, are ascribed to the different temperature and not to the thickness or the structure of the InGaN layers. This is supported by the fact that the damage build-up curve does agree well with the data from Kucheyev et al. ${ }^{14}$ at room temperature, pointing out the relevant role of dynamic annealing processes.

Fig. 2a shows the XRD 20/ $\omega$ scans for the 0002 reflection of the sample before and after the implantation (corresponding to a total fluence of $4 \cdot 10^{16} \mathrm{~cm}^{-2}$ and named asimplanted) as well as the fits to these curves. The spectrum of the as-grown sample is well described considering the nominal structure without any roughness or deformation (Debye-Waller factor of $\sim$ ) but allowing a slight variation of individual layer thicknesses below $0.5 \mathrm{~nm}$. The as-grown sample shows up to 13 superlattice peaks, in good agreement with the simulated structure, reflecting excellent crystal and interface quality. After the implantation, a clear broadening of the main peaks is observed and several superlattice peaks are missing as a result of the damage build-up. The fit assumed the same SL structure as the as-grown sample but allowing slightly higher variation of layer thicknesses (up to $0.7 \mathrm{~nm}$ deviation from the nominal structure) and a decreased Debye-Waller factor $(\sim 0.5$ for the first three periods increasing until reaching again unity for the deeper layers). Furthermore, a deformation of the implanted layers was taken into account yielding a perpendicular strain of maximum $\sim 1 \%$ for the three first periods and decreasing to zero for the deeper unimplanted layers. 
The in-plane $\left(\mathrm{Q}_{\mathrm{x}}\right)$ and out-of-plane $\left(\mathrm{Q}_{\mathrm{z}}\right)$ reciprocal lattice points were obtained from the corresponding $(10 \overline{1} 5)$ reciprocal space maps of both samples, shown in Fig. 2b-c. In addition to the broadening, the maps confirm the strong suppression of the SL peaks (the remaining peak above $\mathrm{GaN}$ being due to the $\mathrm{K}_{\alpha 2}$ contribution from the monochromator). The elongated peak below $\mathrm{GaN}$ is associated to the expansion of the $c$-lattice parameter in the heterostructure, an effect well-documented for implantation in thin nitride films ${ }^{19,20}$. It should be noted that the dynamical theory may not be well suited to describe XRD of the highly damaged sample after high fluence implantation. Nevertheless, the results are in good agreement with the RBS and TEM results presented in this paper suggesting a deterioration of the crystal quality, in particular of the first three periods, while intermixing remains below the detection limits of the employed techniques.

Fig. 3 shows TEM images of the defects formed in the as-implanted maximum-fluence sample. Dark field images, recorded using 0002, 1-120 and 1-100 reflection $g$ vectors, respectively (Fig. 3a-c), reveal a heavily damaged surface region. Fig. 3a, taken under the $g=0002$ condition, indicates the presence of a large number of point defect clusters, interstitials or vacancies, also confirmed in Fig. 3b. Fig. 3c, exciting the $g=1-100$ reflection, shows that, in addition to the defect clusters, a complex network of basal stacking faults (BSFs), both intrinsic ( $\mathrm{I}_{1}$ and $\mathrm{I}_{2}$ type) and extrinsic (E), and prismatic stacking faults $(\mathrm{PSFs})^{21,} 22$ has been generated during the implantation. A high resolution example of one $\mathrm{I}_{2}$ BSF inside the implanted region is exhibited in Fig. 3d. The formation of these extended defects agrees well with previous reports on both $\mathrm{GaN}^{15,19}$ and $\mathrm{InGaN}^{23}$, where the clusters and planar defects have been pointed out for relatively high implantation fluences. A similar defect structure of the ternary and 
binary compounds can explain the homogenous distribution of defects observed in the present case, where the presence of the QWs does not seem to affect the defect distribution and the migration/annihilation properties.

Fig. 4 shows HAADF micrographs of (a) the as-grown and (b) the as-implanted samples. After the implantation, the top first QWs are not clearly distinguishable due to the high damage levels, which are known to distort the unit cell either by accumulation of point defects or strain fields associated with extended defects, both cases disimprove on the contrast of HAADF-STEM images ${ }^{24,25}$, and can render the direct interpretation of contrasts in terms of chemical compositions ineffective. However, local EDX spectra reveal that most of the In remains close to the original QW structure (not shown). This fact was confirmed by grazing incidence $\left(60^{\circ}\right.$ tilt between the surface normal and the incoming beam) RBS measurements, which clearly resolve the first two InGaN QWs which are completely separated in the spectrum. Fig 4c directly compares the RBS spectra of the as-grown and as-implanted sample; no major difference can be discerned. Both spectra are compatible with the targeted original depth profile. Nevertheless, some degree of intermixing cannot be completely excluded due to the limited depth resolution of the technique. This result differs from those obtained for AlN/GaN MQWs where a partial intermixing can be induced by $100 \mathrm{keV} \mathrm{Ar}^{+}$implantation ${ }^{8}$. This fact might be related to the different efficiency of the ballistic intermixing in both cases (which is expected to be 3 times lower in the current study due to the lower energy and ion mass) but other factors such as the electronic energy-loss contribution, the stress state of the material, and the solubility limits of the elements cannot be ruled out and could also play a role in this regard. 


\section{Conclusions}

We have demonstrated that InGaN/GaN MQWs exhibit a high resistance to ion beam induced mixing at room temperature. Damage builds up with a similar trend for In and Ga atoms, leading to the formation of defect clusters and extended planar defects. Such defects are homogeneously distributed throughout the MQWs, but despite the high damage level attained both EDX-STEM and grazing incidence RBS confirmed that compositional intermixing in the QWs remains low.

\section{Acknowledgements}

We thank Dr. P. Ruterana for fruitful discussions and suggestions. We acknowledge support by FCT Portugal (bilateral project DAAD/FCT 2011-2012, PTDC/FISNAN/0973/2012, SFRH/BPD/74095/2010, Investigador FCT) and Juan de la Cierva program (under contract number JCI-2012-14509). TEM experiments were carried out in the Electron Microscopy -DME- and Sample Preparation -LPM- Divisions of the Central Services of Science and Technology of the University of Cádiz (SCCYT-UCA), being financed by the projects MAT2010-15206 (CICYT, Spain), EU-COST Action MP0805, and P09-TEP-5403 (Junta de Andalucía with EU-FEDER participation). 


\section{REFERENCES}

1 D. A. B. Miller, D.S. Chemla, T.C. Damen, A.C. Gossard, W. Wiegmann, T.H. Wood and C.A. Burrus, Phys. Rev. Lett. 53, 2173 (1984).

2 K. P. O’Donnell, M. Auf der Maur, A. Di Carlo, K. Lorenz, and the SORBET consortium, Phys. Stat. Sol. RRL 2, 49 (2012).

3 I. P. Jain, G. Agarwal, Surf. Sci. Rep. 66, 77 (2011).

4 J. Ralston, G.W. Wicks, L.F. Eastman, B.C. De Cooman, and C.B. Carter, J. Appl. Phys. 59, 120 (1986).

5 H. H. Tan, C. Jagadish, J.S. Williams, J. Zou, D.J.H. Cockayne, and A. Sikorski, J. Appl. Phys. 77, 87 (1995).

6 K. J. Beernink, D. Sun, D. W. Treat, and B. P. Bour, Appl. Phys. Lett. 66, 3597 (1995).

7 J. A. Davis, L.V. Dao, X. Wen, C. Ticknor, P. Hannaford, V.A. Coleman, H.H. Tan, C. Jagadish, K. Koike, S. Sasa, M. Inoue and M Yano, Nanotechnology 19, 055205 (2008).

8 A. Redondo-Cubero, K. Lorenz, E. Wendler, D. Carvalho, T. Ben, F.M. Morales, R. García, V. Fellmann and B. Daudin, Nanotechnology 24, 505717 (2013).

9 C. Wetzel, T. Salagaj, T. Detchprohm, P. Li, and J. S. Nelson, Appl. Phys. Lett. 85, 866 (2004).

${ }^{10}$ B. Breeger, E. Wendler, W. Trippensee, Ch. Schubert, W. Wesch, Nucl. Instr. and Meth. B 174, 199 (2001).

11 J. F. Ziegler, J.P. Biersack, U. Littmark The stopping and range of ions in solids (Pergamon Press, New York, 1985).

12 W. J. Bartels, J. Hornstra, D. J. W. Lobeek, Acta Cryst. A42, 539 (1986). 
13 A. Boulle, A. Debelle, J. Appl. Cryst. 43, 1046 (2010).

${ }^{14}$ S. O. Kucheyev, J.S. Williams, J. Zou, and C. Jagadish, J. Appl. Phys. 95, 3048 (2004).

15 P. Ruterana, B. Lacroix, and K. Lorenz, J. Appl. Phys. 109, 013506 (2011).

16 E. Wendler, W. Wesch, E. Alves, A. Kamarou, Nucl. Instr. and Meth. B 218, 36 (2004).

17 N. Hecking, K.F. Heidemann and E. Tekaat, Nucl. Instr. and Meth. B 15, 760 (1986).

18 E. Wendler, Nucl. Instr. and Meth. B 267, 2680 (2009).

19 B. Lacroix, S. Leclerc, A. Declémy, K. Lorenz, E. Alves and P. Ruterana, EPL 96, $46002(2011)$.

20 S. Leclerc, B. Lacroix, Declémy, K. Lorenz, and P. Ruterana, J. Appl. Phys. 112, $073525(2012)$.

21 P. Ruterana and G. Nouet, Physica Status Solidi B-Basic Research 227, 177 (2001).

${ }^{22}$ F. Gloux, T. Wojtowicz, P. Ruterana, K. Lorenz, and E. Alves, Journal of Applied Physics 100, 073520 (2006).

${ }^{23}$ S. O. Kucheyev, J.S. Williams, J. Zou, S.J. Pearton, Y. Nakagawa, Appl. Phys. Lett. 79, $602(2001)$

${ }^{24}$ V. Grillo, E. Carlino, and F. Glas, Physical Review B 77 (2008).

${ }^{25}$ V. Grillo, K. Mueller, K. Volz, F. Glas, T. Grieb, and A. Rosenauer, 17th International Conference on Microscopy of Semiconducting Materials 2011, 326 (2011) 
TABLES

Table 1 - Main parameters obtained from the Hecking model.

\begin{tabular}{lrr}
\hline \hline Parameter & In (w1) & Ga (w2) \\
\hline $\mathbf{P}_{\mathbf{p d}}\left(\mathbf{1 0} \mathbf{w}^{-16} \mathbf{c m}^{\mathbf{2}}\right)$ & $4.5(5)$ & $2.9(5)$ \\
$\mathbf{R}_{\mathbf{p d}}\left(\mathbf{1 0}^{-\mathbf{1 4}} \mathbf{c m}^{\mathbf{2}}\right)$ & $1.5(2)$ & $1.2(2)$ \\
$\mathbf{C}_{\mathbf{p d}}\left(\mathbf{1 0}^{-16} \mathbf{c m}^{\mathbf{2}}\right)$ & $1.2(2)$ & $0.4(2)$ \\
$\mathbf{P}_{\mathbf{a}}\left(\mathbf{1 0}^{-18} \mathbf{c m}^{\mathbf{2}}\right)$ & $1(1)$ & $1(1)$ \\
$\mathbf{G}_{\mathbf{a}}\left(\mathbf{1 0}^{-17} \mathbf{c m}^{\mathbf{2}}\right)$ & $4(1)$ & $8(1)$ \\
\hline
\end{tabular}




\section{FIGURE CAPTIONS}

Figure 1. (Color online) (a) RBS/C spectra for different $\mathrm{N}^{+}$fluences (note the semi$\log$ scale). The surface energy positions for In and Ga are marked for better identification of the elements. Energy windows used for the individual analysis of damage are marked as $\mathrm{w}_{1}(\mathrm{In})$ and $\mathrm{w}_{2}(\mathrm{Ga})$. Fluence values are given in $\mathrm{cm}^{-2}$. (b) Damage level for window $\mathrm{w}_{1}$ (In) and $\mathrm{w}_{2}(\mathrm{Ga})$ as a function of the fluence. The corresponding fits using Hecking's model are also shown (lines). Results for thin InGaN films implanted with Au at room and low temperature are also shown as a reference (adapted from refs. ${ }^{14,16}$ ).

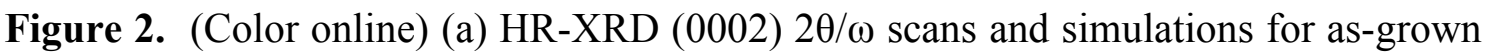
and as-implanted $\left(4 \cdot 10^{16} \mathrm{~cm}^{-2}\right)$ MQWs. (b,c) Reciprocal space mappings for both samples. The color bar represents intensity in a log scale.

Figure 3. (Color online) Dark field TEM images of the as-implanted sample $\left(4 \cdot 10^{16}\right.$ $\mathrm{cm}^{-2}$ ) acquired with $\mathrm{g}=0002(\mathrm{a}), \mathrm{g}=1-120(\mathrm{~b})$, and $\mathrm{g}=1-100$ (c) orientations, marked in red. (d) HR-TEM image corresponding to the implanted region, where an $\mathrm{I}_{2}$ intrinsic $\mathrm{BSF}$ is visible.

Figure 4. (Color online) HAADF micrographs showing the as-grown (a) and the asimplanted (b) sample. (c) Grazing incidence $\left(60^{\circ}\right)$ RBS spectra of as-grown and as-implanted sample. The simulation for the nominal structure is also show for comparison. Markers for the surface energies of In and Ga are displayed. 

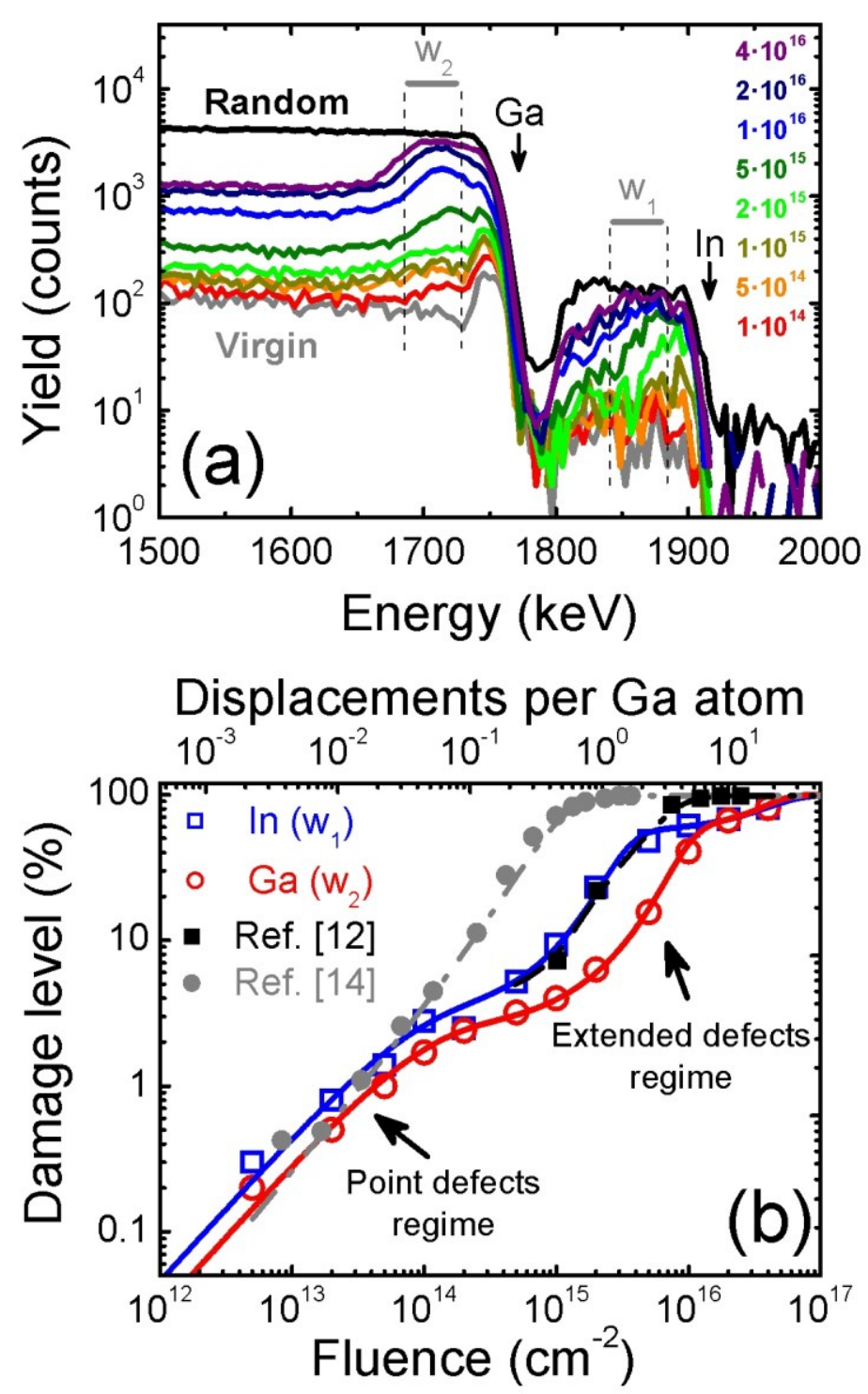

Figure 1 - Redondo-Cubero et al. 

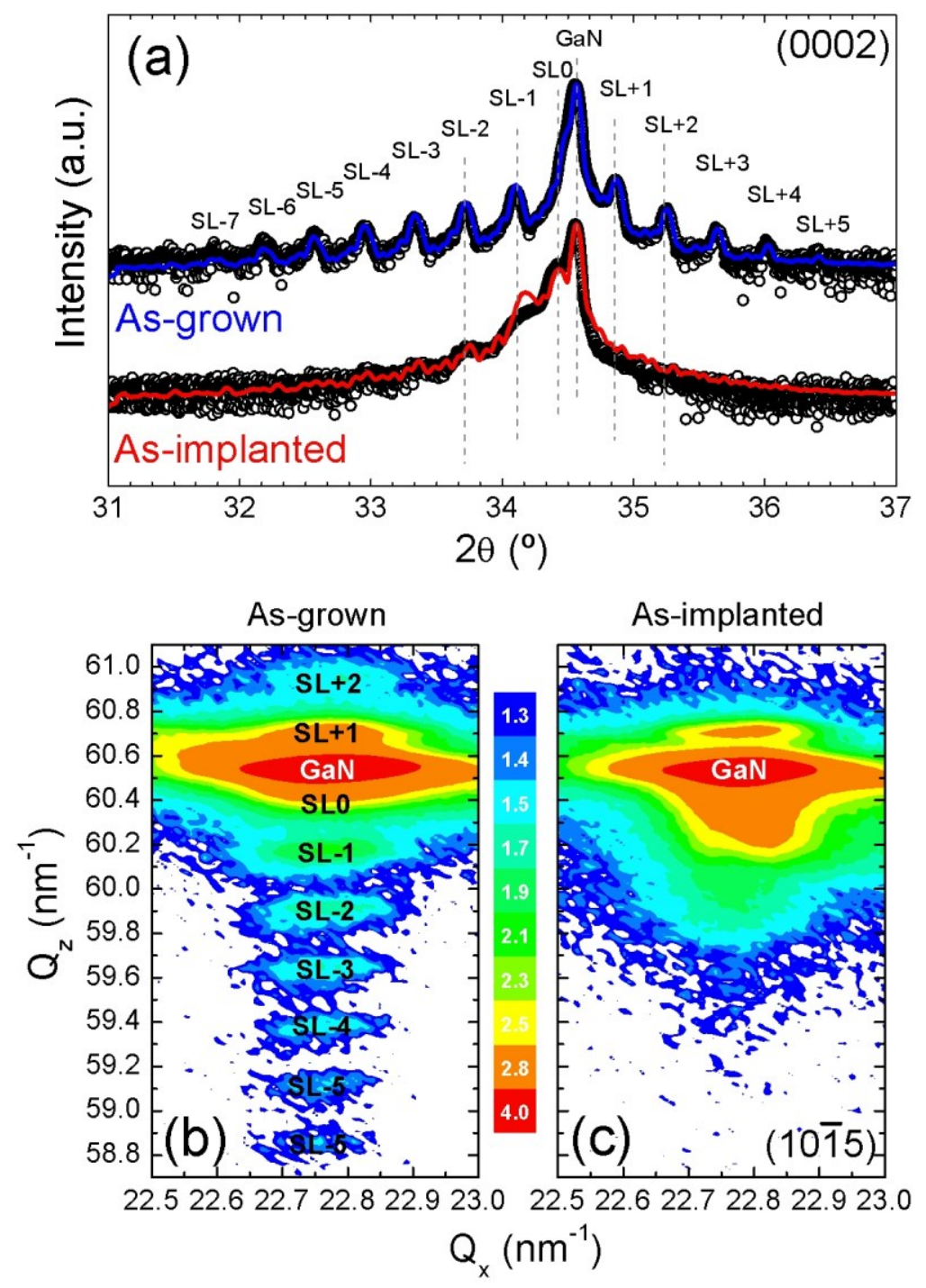

Figure 2 - Redondo-Cubero et al. 

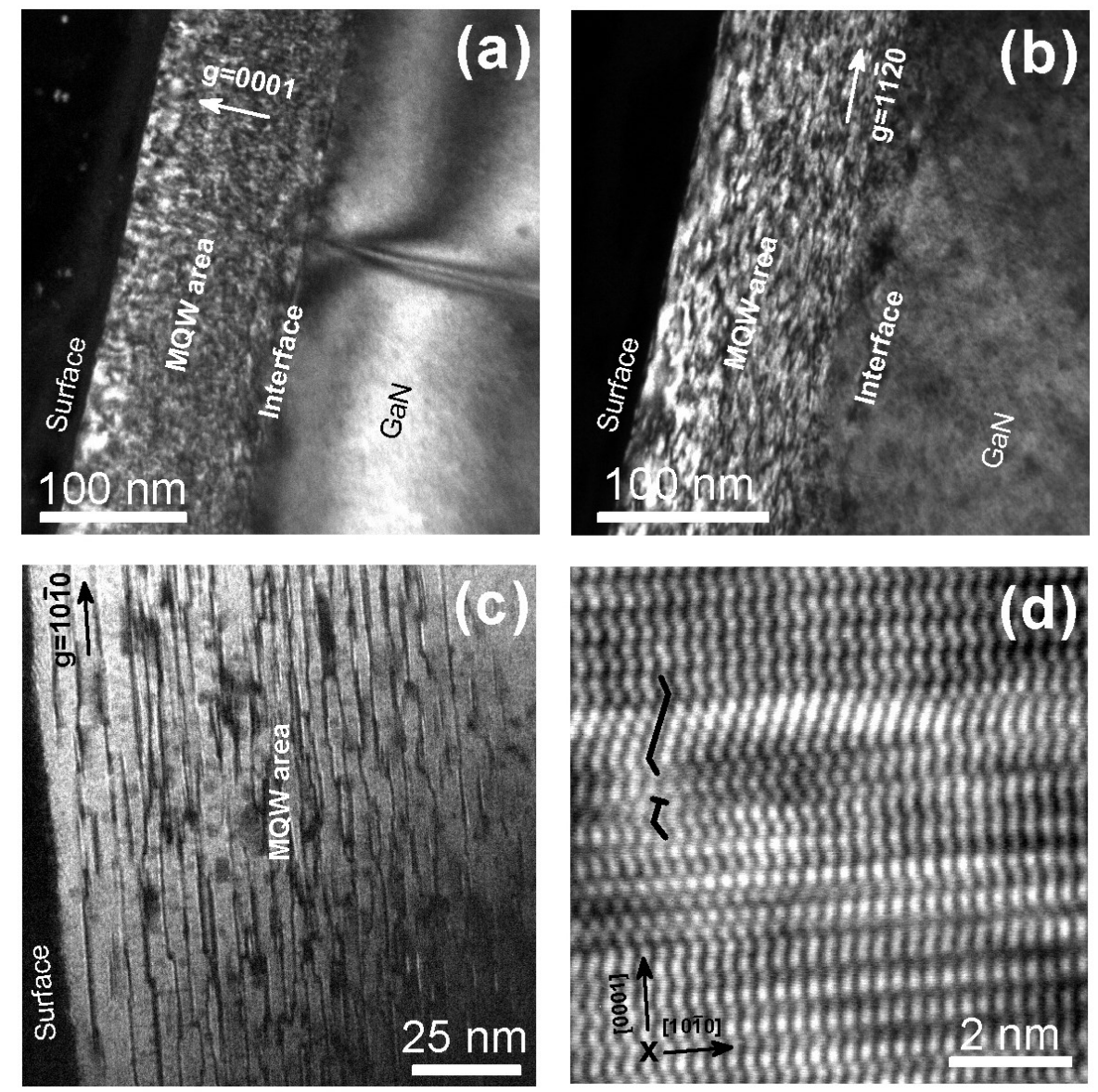

Figure 3 - Redondo-Cubero et al. 

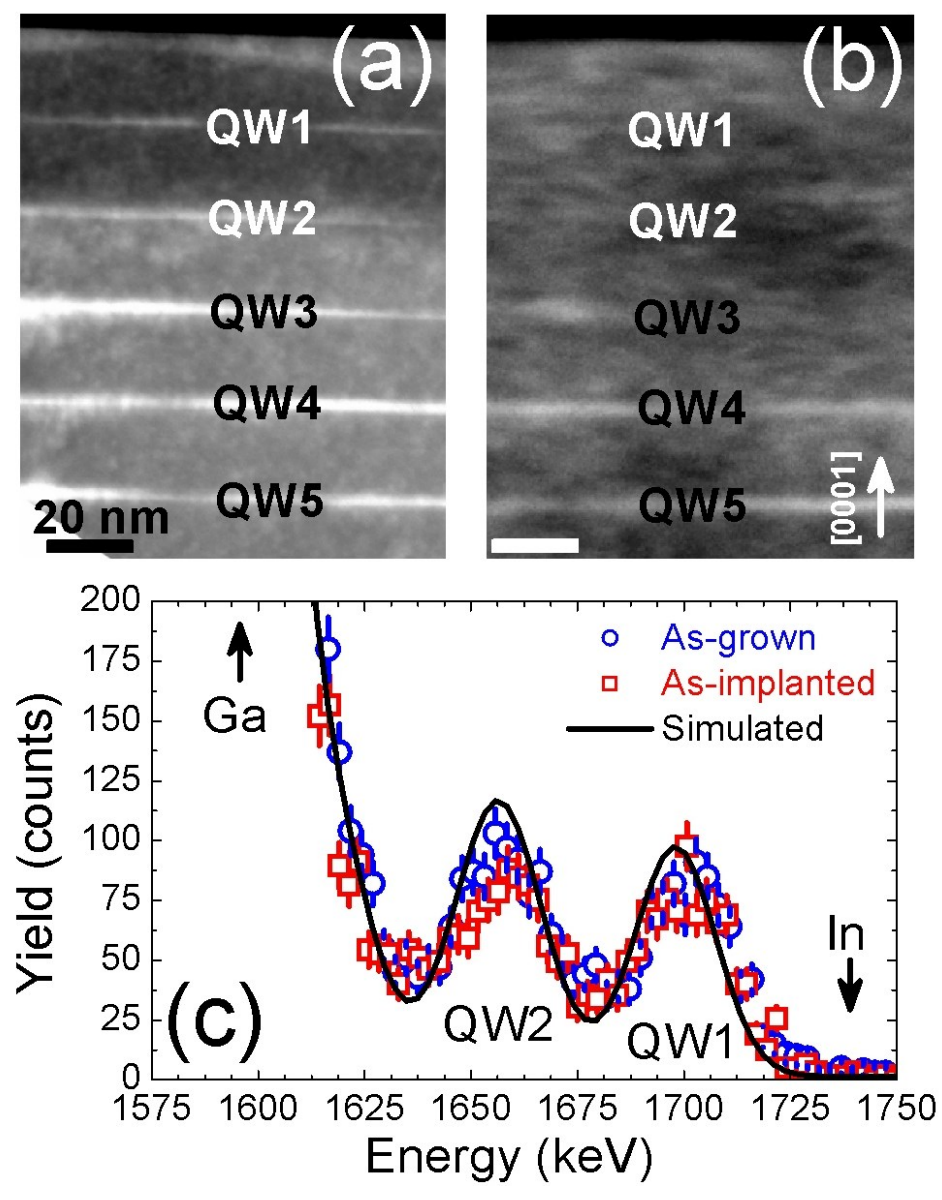

Figure 4 - Redondo-Cubero et al. 